\title{
Reflets
}

Revue ontaroise d'intervention sociale et communautaire

\section{Les décisions de recherche d'emploi des nouvelles bachelières en sciences infirmières}

\section{Sylvie Larocque et Suzanne Caty}

Volume 5, numéro 2, automne 1999

La santé des francophones de l’Ontario

URI : https://id.erudit.org/iderudit/026280ar

DOI : https://doi.org/10.7202/026280ar

Aller au sommaire du numéro

Éditeur(s)

Reflets : Revue ontaroise d'intervention sociale et communautaire

ISSN

1203-4576 (imprimé)

1712-8498 (numérique)

Découvrir la revue

Citer cet article

Larocque, S. \& Caty, S. (1999). Les décisions de recherche d'emploi des nouvelles bachelières en sciences infirmières. Reflets, 5(2), 225-233.

https://doi.org/10.7202/026280ar

Tous droits réservés (C) Reflets : Revue ontaroise d'intervention sociale et communautaire, 1999
Ce document est protégé par la loi sur le droit d'auteur. L'utilisation des services d'Érudit (y compris la reproduction) est assujettie à sa politique d'utilisation que vous pouvez consulter en ligne.

https://apropos.erudit.org/fr/usagers/politique-dutilisation/ 


\section{Les décisions de recherche d'emploi des nouvelles bachelières en sciences infirmières $^{1}$}

Sylvie L arocque, inf. M Sc.

Suzanne C aty, inf. M Sc.

É coles des sciences infirmières U niversité $L$ aurentienne, Sudbury, 0 ntario

\section{Introduction}

Le marché du travail en soins infirmiers a subi plusieurs changements au cours des dernières années. Les possibilités d'emplois pour les nouvelles diplômées avaient diminué énormément en raison de la restructuration du système de santé. De plus, cette restructuration a occasionné des pertes d'emplois, la diminution du nombre de lits, la fermeture de certains hôpitaux et un accroissement des postes à temps partiels ou occasionnels. Toutes ces transformations, combinées à une bai sse d'inscriptions dans les programmes de sciences infirmières rendent inévitable une pénurie prochaine d'infirmières (N oesgaard 1998; Sibbald 1998). R éagissant à cette urgence, le gouvernement de l'O ntario a annoncé l'injection de fonds supplémentaires pour créer des postes additionnels en soins infirmiers (Gouvernement de l'O ntario 1999). 
Par le passé, les diplômées en sciences infirmières qui étudiaient dans le nord ontarien avaient aussi tendance à choisir cette région comme lieu de pratique. Étant donné la situation précaire d'emplois en sciences infirmières, nous avons voulu connaître l'impact des changements dans le système de santé sur la capacité de nos diplômées de faire carrière dans leur domaine d'études. À cette fin, en 1994, nous avons débuté un projet de recherche ayant pour but d'examiner les stratégies de recherche d'emploi utilisées par nos diplômées du programme de formation initiale (français et anglais) et les facteurs qui l'influencent ${ }^{2}$. C e projet a été approuvé par le comité d'éthique de I'U niversité Laurentienne. $\mathrm{N}$ ous avons procédé au moyen d'une enquête par questionnai re auprès d'anciennes diplômées du programme de sciences infirmières de I'U niversité Laurentienne afin d'obtenir de l'information sur leur décision d'emploi en soins infirmiers (Larocque et Caty 1997a, 1997b, 1997c; C aty et Larocque 1998, 1999b).

Le présent article fera un résumé des résultats de notre recherche auprès des diplômées qui ont étudié en français. Plus particulièrement, nous présenteronsun résumé des donnéessociodémographiques, du profil d'emploi de ces diplômées, desfacteurs qu'elles ont jugés importants dans leurs décisions d'emploi et des stratégies mises en œuvre pour affronter un marché de travail difficile.Toutesles diplômées du programme francophone de 1995, 1996 et 1997 ont été invitées à participer au projet de recherche. $\mathrm{N}$ otre échantillon initial comprenait 30 diplômées. Vingt-deux $(73,3 \%)$ ont rempli le questionnaire. De ce nombre, nous en avons retenu 21 pour procéder à l'analyse des données, car une des diplômées qui a retourné le questionnaire a indiqué qu'elle ne travaillait pas présentement en raison d'un congé de maternité. Son questionnaire a été exclu de l'analyse car plusieurs sections étaient incomplètes. 


\section{Données socio-démographiques et profil de carrière professionnel}

L'âge moyen des diplômées était de 24.5 ans. H uit (38,1 \%) étaient mariéeset douze $(57,1 \%)$ célibatai res. Ellesétaient tousdirectement ou indirectement impliquées dans les soins infirmiers. La majorité $(61,9 \%)$ travaillait dans des centres hospitaliers de soins aigus (Tableau 1). Plus des trois quarts (76,2\%) disaient travailler à temps partiel ou occasionnellement, car il n'y avait pas de postes à temps plein. Près de la moitié $(47,6 \%$ ) détenait plus d'un emploi afin de survivre financièrement et d'acquérir plus d'expériences.

Seulscinq diplômées $(23,8 \%$ ) travaillai ent à temps plein. Parmi cescinq, troistravaillaient en milieu communautaire, une, en sécurité au travail et l'autre, dans un centre hospitalier de soins aigus hors du C anada. La majorité (76,2 \%) des diplômées travaillaient dans le nord ontarien, dont deux seulement détenaient un emploi à temps plein. Chez les autres diplômées, trois travaillaient dans le sud de I'O ntario, une, au Q uébec et une, à l'extérieur du C anada.

\section{Tableau 1 - Milieux de pratique des diplômées}

\begin{tabular}{|l|c|}
\hline Milieu de pratique & Nombre de diplômées $\mathbf{~} \mathbf{~ ( n = 2 1 ) ~}^{\text {No }}$ \\
\hline C entre hospitalier de soins aigus & 13 \\
\hline Service de soins à domicile & 4 \\
\hline Santé publique & 4 \\
\hline Autres agences communautaires & 3 \\
\hline Centre d'accueil pour personnes âgées & 2 \\
\hline Bureau de médecin & 2 \\
\hline Centre de soins prolongés & 1 \\
\hline Service de santé au travail & 1 \\
\hline Centre de soins psychiatriques & 1 \\
\hline
\end{tabular}

${ }^{1} \mathrm{C}$ ertaines avaient deux milieux de pratique 


\section{Sentiments éprouvés}

Lors de leur recherche d'emploi, les sentiments éprouvés par les diplômées tenaient davantage de l'optimisme et de l'espoir. C ependant, certaines ont aussi indiqué avoir fait l'expérience de sentiments négatifs tels la colère et le désespoir.

\section{Facteurs considérés}

N ous avons demandé aux diplômées d'évaluer l'importance de certains facteurs ayant un impact sur leur recherche d'emploi en soins infirmiers. Ces facteurs étaient:

- leurs objectifs de carrière en soins infirmiers à court terme;

- leurs objectifs de carrière en soins infirmiers à long terme;

- leur connaissance de la réalité présente du marché de travail;

- leur connaissance des nouvellesopportunités de carrières dans la profession infirmière;

- l'impact causé par les changements au sein du système des soins de la santé sur la pratique, sur leur programme de formation et sur l'accessibilité des services pour les clients;

- leur salaire potentiel d'emploi en soins infirmiers;

- leur situation financière face à l'impossibilité de trouver un emploi en soins infirmiers;

- leurs valeurs culturelles;

- leurs responsabilitésfamiliales,

- leur réseau social;

- leur style de vie;

- leur flexibilité de déménager afin de trouver un emploi en soins infirmiers;

- leur désir de déménager afin de débuter une carrière en soins infirmiers;

- leur refus de déménager afin de débuter une carrière en soins infirmiers. 
En général, la majorité des facteurs a été considérée comme important dans leur recherche d'emploi en soins infirmiers. Toutefois, certains facteurs se distinguent plus que d'autres. A insi, trois facteurs ont été perçus comme étant très importants, soit leur connaissance actuelle de la réal ité du marché de travail, leurs objectifs de carrière en soins infirmiers à court et à long terme. $\grave{A}$ l'opposé, trois facteurs ont été perçus comme ayant moins d'importance, soit le refus de déménager, leurs réseaux sociaux et leurs valeurs culturelles.

\section{Stratégies utilisées}

Parmi une liste de stratégies proposée, les diplômées devaient choisir cellesqu'ellesont utilisées pour tenter d'intégrer un marché du travail difficile en soinsinfirmiers Lescinq stratégiesutilisées le plussouvent étaient : l'envoi d'un curriculum vitae à plusieurs agences de soins, d'être flexible dansla recherche d'un emploi, d'accepter la possibilité qu'elles ne puissent pas obtenir un emploi à temps plein, d'accepter le fait qu'ellesne puissent pas exercer leur profession dansleur domaine préféré et d'être prête d'accepter n'importe quel emploi en soins infirmiers. Par ailleurs, la stratégie la moins souvent utilisée était celle d'attendre ses résultats aux examens de pratique avant de chercher un emploi en soins infirmiers.

Plusieurs diplômées ont fait des commentaires personnels à la fin de leur questionnaire. Certaines jugent positivement leurs expériences de recherche d'emploi en soins infirmiers, comme en témoignent ces quelques commentaires:

E $n$ dépit de tout, je me considère quand même assez chanceuse [... . D epuis ma graduation, j'ai eu l'occasion de travailler comme infirmière - il y avait seulement deux mois où j'avais rien - mes amies ont rencontré plusieurs difficultés..

II faut être flexible envers les heures de travail (accepté l'emploi même si c'est seulement sur appel) [... ] j'ai 
trouvé cela plus facile (la recherche de travail) après un peu de mois d'expérience [...] mais il faut souvent quitter sa famille et déménager.

Je crois que je suis une exception en terme de mon expérience pour trouver un emploi parce que j'ai été embauché seulement que deux semaines après avoir présenté mon $C . V$.

D 'autres diplômées ont mentionné l'aide et l'appui reçus de leur employeur:

$M$ on agence a assisté avec les finances pour prendre un certificat en soins intensifs et ils procurent une orientation spédale pour que je puisse ensuite travailler pour eux dans ce domaine

[... ] la découverte d'un mentor qui m'encourage vers le leadership et la gestion.

Enfin, certaines ont tenu à souligner la persévérance nécessaire pour travailler en soinsinfirmiers et pour surmonter leur situation financière précaire:

L es prêts accordés pour les études deviennent difficiles à repayer suite à la graduation. II faut persister pour de l'emploi pour pouvoir remettre l'argent à R A F FE 0 .

\section{Conclusion}

De 1995 à 1997, les résultats de cette recherche démontrent que les diplômées étaient au courant de la situation difficile du marché de travail en soinsinfirmiers. Elles ont mentionné plusieursfacteurs ayant un impact dans leur recherche d'emploi et plusieursstratégies mises en œuvre afin de les aider dans leur recherche d'emploi. Si toutes ont été capables de trouver un emploi, la majorité de ces emplois était à temps partiel ou occasionnel. C ette tendance du marché du travail en soins infirmiers est un phénomène bien connu (Bookey-Bassett 1998). La majorité des diplômées du 
programme francophone est demeurée dans le nord de l'O ntario pour y débuter leur carrière d'infirmière, bien que seulement deux d'entre elles se soient trouvées un emploi à temps plein. Cet élément tend à démontrer l'importance de l'appartenance régionale, en particulier au $\mathrm{N}$ ord, dans la décision d'y débuter sa carrière. D u moins, est-ce un élément qui vaudrait la peine d'être étudié dans une prochaine étude? II est aussi possible que le bilinguisme des diplômées francophones ait été un atout dans leur recherche d'emploi en soins infirmiers. II importe de noter que plusieurs des diplômées du programme anglophone ont débuté leur carrière d'infirmière aux États- $U$ nis afin d'obtenir un emploi à temps plein. U ne telle avenue n'est pas aussi évidente chez les francophones s'ils veulent conserver leur appartenance culturelle (C aty et Larocque 1999).

La profession s'inquiète de la tendance vers des emplois à temps partiel ou occasionnels et de son impact sur la sécurité des soins offerts aux clients et sur le développement d'une carrière professionnelle (Grinspun 1998; Shamian 1998). De plus, ce type d'emploi nuit à la capacité de nos diplômées d'atteindre une certaine sécurité financière (H iscott 1996). C ette insécurité financière était le lot de plusieurs de nos diplômés, car près de la moitié devaient avoir deux emplois afin de gagner un salaire décent.

La profession infirmière à travers ses représentantes (R N AO $\mathrm{N}$ ews 1998) a transmis au ministère de la Santé de l'O ntario, ces inquiétudes concernant le processus de restructuration, la réduction du personnel en soins infirmiers, le nombre élevé d'emploi occasionnel et à temps partiel. Selon elles, ces transformations affectent grandement la capacité d'offrir des soins infirmiers de qualité et sécuritaire. Suite à cette sortie, le M inistère de la santé (1998) a décidé de créer un groupe de travail pour étudier l'impact de la restructuration des services de santé sur les soins infirmiers en 0 ntario. Cette décision démontre que le gouvernement reconnaissait alors le bien-fondé des inquiétudes de la profession infirmière. Plus récemment encore, le $G$ roupe de travail (1999) et l'O rdre des infirmières et infirmiers de l'O ntario (Boyce 1999) ont déposé leurs principales recommandations. O n 
y mentionne, entre autres, le besoin d'augmenter le nombre d'emploi à temps plein et à temps partiel et de mettre fin aux coupures d'emploi en soins infirmiers.

C es recommandations ont été acceptées intégralement par le gouvernement de l'O ntario. Ainsi, le gouvernement s'engage à subventionner 12000 nouveaux postes au cours de la prochaine année (Gouvernement de l'O ntario 1999). L'augmentation de postes, surtout ceux à temps plein, ne peut qu'améliorer les conditions de travail des infirmières et la qualité des soins. C ela facilitera aussi l'intégration au marché du travail des nouvelles diplômées et est propre à favoriser une meilleure sécurité d'emploi et la possibilité de poursuivre leur plan de carrière en soins infirmiers. R este à voir si les promesses d'augmenter les postes à temps plein et à temps partiel se concrétiseront dans un avenir rapproché. La profession devra être vigilante et vérifier si le gouvernement tient ses promesses.

C ela dit, malgré les décisions récentes du gouvernement concernant les nouveaux postes en soins infirmiers, la situation d'emploi demeure précaire et évolue constamment. C'est pourquoi nous nous proposons de suivre périodiquement nos diplômées afin de connaître leurs décisions d'emploi.

\section{Bibliographie}

BO O KEY-BASSETT (1998). «C areer planning concerns of undergraduate nursing students and new graduates», R egistered N urse Journal, vol. 10, no 3, 8-9.

BOY CE, C. (1999). «R apport du Groupe de travail sur les soins infirmiers: le ministère consulte I'O IIO ». C ommuniqué de l' 0 rdre des infirmières et infirmiers de l' 0 ntario, vol. 24, no 1, 26- 27.

CATY, S. et S. LARO C Q U E (1999a). T he 1997 BsdV G eneric N ursing G raduates' J ob Search A ctivities and E mployment Profil, Sudbury, C entre for R ural and $\mathrm{N}$ orthern $\mathrm{H}$ ealth R esearch, Laurentian U niversity.

CATY, S. et S. LAR O CQ U E (1999b). N ew BsdV graduates: W here are they pradising? A comparative analysis of three classes of $G$ raduates $(1995,1996,1997)$, Sudbury, C entre for $R$ ural and $N$ orthern $H$ ealth $R$ esearch, Laurentian U niversity.

CATY, S. et S. LAR O C Q U E (1998). The 1996 B sdV G eneric N ursing G raduates' Job Search A ctivities and E mployment Profile, Sudbury, Centre for R ural and N orthern H ealth R esearch, Laurentian U niversity. 
GOVER N M EN T OF ONTAR IO (1999, M arch 19). H arris Exceeds Task Force R ecommendation : A nnounces $12,000 \mathrm{~N}$ ew N urses, Press $\mathrm{R}$ elease, Toronto.

GR IN SPU N , D. (1998). «Speaking out for health, speaking out for nursing : N urses voices must be heard », R egistered N urse Journal, vol. 10, no 2, 16-17.

HISC OT T, R .D. (1996). «D ebt profiles of new grads», T he $C$ anadian N urse, vol. 92, no 3, 32-37.

LARO C Q U E, S. et S. CATY, (1997a). «G raduating nursing students' initial career plans: Influencing factors and responses », The C anadian N urse/ L'infirmière canadienne, vol. 93, no 10, 31-35.

LAR O CQ U E, S. et S. CATY (1997b). «N ursing graduates career activities:A one-year follow-up study », R egistered N urses Journal, vol. 9, no 5, 5-7.

LAR O CQ U E, S. et S. CATY (1997c). T he 1995 B sdV generic nursing graduates job search adivities and employment profile, N H H H RU Working Paper Series, Sudbury, Laurentian U niversity.

N O ESGAAR D, C. (1998). «T he cost of caring », R egistered N urse Journal, vol. 10, no 1, 4.

ON TAR IO M IN IST RY OF HEALTH (1999). G ood N ursing, G ood $\mathrm{H}$ ealth: A n Investment for the $21^{\text {tt }} \mathrm{C}$ entury : R eport of the $\mathrm{N}$ ursingTask Force Toronto, Q ueen's Printer for $\mathrm{O}$ ntario.

R N AO N ews (1998). "N ursing organisations issue position statement on nursing in O ntario ", R egistered N urse Journal, vol. 10, no 4, 20.

SH AM IAN , J. (1998). «Keep the pressure on », R egistered N urse Journal, vol. 10, no 4, 4.

SIBBALD, B. (1998). «T he future supply of registered nurses in C anada », The $C$ anadian $N$ urse, vol. 94, no $1,22-23$.

\section{Notes}

1. A fin d'alléger le texte, le féminin est employé comme terme générique.

2. Ce projet fait partie du programme de recherche du C entre en santé dans les milieux ruraux et du N ord de I'U niversité Laurentienne, Sudbury. 